\title{
Nonlinear damping in energy harvesters driven by colored noise
}

\author{
M. Romero-Bastida and S. Cholula-Ramirez \\ Sección de Estudios de Posgrado e Investigación Escuela Superior de Ingeniería \\ Mecánica y Eléctrica-Culhuacán, Instituto Politécnico Nacional, \\ Avenida Santa Ana 1000, Colonia San Francisco Culhuacán, Delegación Coyoacan, Distrito Federal 04430, Mexico. \\ e-mail:mromerob@ipn.mx
}

Received 26 April 2018; accepted 3 August 2018

\begin{abstract}
We study the performance of an electromechanical oscillator as an energy harvester driven by finite-bandwidth random vibrations under the influence of both a stiffness-type nonlinearity and a nonlinear damping that has recently been found to be relevant in the dynamics of submicrometer mechanical resonators. The device was numerically simulated and its performance assessed by means of the net electrical power and the efficiency of the conversion of the supplied power by the noise into electrical power for exponentially correlated noise. We tune the parameters to achieve a good performance of the device for non-negligible amplitudes of the nonlinearity of the oscillator and the damping.
\end{abstract}

Keywords: Energy harvesting; nonlinear friction; wide spectrum.

PACS: 05.40.Ca; 05.10.Gg; 46.65.+g; 84.60.-h

\section{Introduction}

Energy harvesting is the conversion of ambient energy present in the environment into electrical energy. It is identical in principle to large-scale renewable energy generation, for example, solar or wind power, but very different in scale. While large-scale power generation is concerned with megawatts of power, energy harvesting typically refers to micro- and/or milli-watts, i.e. much smaller power generation systems. The development of energy harvesting has been driven by the proliferation of technologies such as autonomous wireless electronic systems, a classic example being wireless sensor nodes which combine together to form wireless sensor networks; for this type of systems local power supply is an attractive option. By converting ambient energy in the environment the energy harvester can provide the required electric power for the lifetime of the wireless system which is also free to be embedded or placed wherever it is best suited to perform its function. Energy harvesting typically exploit kinetic, thermal, solar, or electromagnetic radiation sources. Thermal gradients can be exploited by using thermoelectric generators whereas solar energy is harvested using photovoltaics. However, the most prominent type of devices are mechanical vibration energy harvesters that convert kinetic energy via electromagnetic, electrostatic, or piezoelectric transduction into electrical energy [1-5].

Mechanical energy harvesters, also known as vibration power generators, are typically, although not exclusively, inertial spring-mass systems where electrical power is extracted by employing one or a combination of different transduction mechanisms. Early studies considered linear springs and harmonic oscillators and treated the external vibrations as sinusoidal vibrations. As most vibration power generators are resonant systems, they generate maximum power when the resonant frequency of the generator matches the ambient vibration frequency, known as resonant energy harvesting [1].
Adaptive generators try to minimize the difference between these two frequencies in order to maximize the amount of generated power $[2,4,6]$. Nearly all current vibration transducers operate in this regime [7]. This approach presents numerous drawbacks, being one of the most important ones that the linear harvester resonant peak is necessarily very narrow [8]. Thus, if the environmental vibration frequency deviates from the harvester resonance very little power is generated. To overcome this limitation various groups have begun to study mass-spring systems with nonlinear springs and nonlinear oscillators [9-12]. Many important results have been obtained if the broadband ambient vibrations are modeled by Gaussian white noise. For example, it has been determined, using the Fokker-Planck equation to describe Duffing-type energy harvesters, that the mean power output of the device is not affected by the nonlinearity of the spring $[13,14]$. Also the upper bounds on the power output of both linear and nonlinear energy harvesters driven by Gaussian white noise have been obtained [15]. The latter ones can be advantageous since the size of the device can be reduced without affecting the power output [14]. Now, while some environmental excitations exhibit the characteristics of broadband white noise, many others have most of their energy trapped within certain frequency bandwidths, i.e. external colored noise. After some early experimental and simulation studies $[12,16]$ the power output of both a monostable [13] and a bistable Duffing oscillator with a symmetric potential [17] driven by Ornstein-Uhlenbeck noise was determined by approximate methods, and the exact analytical expressions for the net electrical power and efficiency of the conversion of the power supplied by exponentially correlated noise into electrical power was derived for a linear electromechanical oscillator employed as an energy harvester [18].

In almost all theoretical models mentioned above, the coupling between the mechanical oscillator and the ambient 
noise were supposed to be linear. This is because the energy dissipation (damping) in previous studied systems were supposed to be linear in general. However, damping has an important impact on the dynamic behavior of submicrometre mechanical resonators, and recent researches have revealed that nanostructures with high aspect ratio such as nanotubes and graphene nanoribbons can be easily driven into nonlinear dissipation regime [19]. This nonlinear damping has been so far studied mainly within the field of thermal transport in a spin-boson nanojunction [20], in nonlinear two-level molecular junctions [21], in a monomodal harmonic molecular junction (a single harmonic oscillator) [22], and in a onedimensional lattice of coupled nearest-neighbor harmonic oscillators [23].

In this work we propose to study the resonator driven by colored noise studied in Ref. [18] with a nonlinear damping between the mechanical oscillator and the source of ambient noise to explore its influence on the performance of the device as an energy harvester. The proposed nonlinear damping has been previously considered within the context of a micromechanical oscillator model in Ref. [19] to properly account for various experimental observations made in mechanical resonators based on carbon nanotubes and graphene sheets, as mentioned above.

The rest of the paper is organized as follows: in Sec. 2 we present the model as well as our methodology. Results for both a linear and nonlinear oscillator are reported in Sec. 3. Finally, in Sec. 4 we discuss the results so far obtained and propose ways to further improve them.

\section{Model and methodology}

The herein considered energy harvester is a device that converts the power supplied by external noise into electrical energy. This process begins with the damped oscillator being driven by the external noise. Its kinetic energy is then converted via a piezoelectric transducer mechanism into electrical energy that is then stored in a capacitor. The mechanical part of the device is described by the equation for the momentum of the stochastically driven damped oscillator of mass $m$, which reads as

$$
\dot{p}+\gamma p+\beta q^{2} p+F_{\text {tran }}(q, V)+\omega_{0}^{2} q+\alpha q^{3}=\eta(t),
$$

with the dot standing for temporal derivative. In this equation $\omega_{0}$ is the natural frequency of the oscillator, $\alpha q^{3}$ is the so-called Duffing force, $\gamma$ is the linear damping coefficient, $\beta q^{2} p$ is the nonlinear damping term, $\eta(t)$ is the random driving force, and $F_{\text {tran }}(q, V)$ is the transducer force due to the motion-to electricity conversion mechanism, which depends on the geometry of the transducer and on how the circuit that implements the energy conversion cycle operates. It opposes to the motion, just as the friction force, and has its origin in the energy loss that occurs when kinetic energy is converted into electric energy. The simplest expression for this function is $F_{\operatorname{tran}}(q, V)=k_{v} V$, where $k_{v}>0$ is a piezoelectric parameter and $V(t)$ is the voltage. The dynamical equation for the voltage has to take into account the load resistance $R_{L}$ and the capacitance $\mathcal{C}$ of the piezoelectric component, as well as the connecting function $F(p, V)$ with the oscillator; all these variables are related by the equation $\dot{V}=F(p, V)-V / \tau_{p}$, where $\tau_{p}=R_{L} \mathcal{C}$ is the time associated to the charging process of the piezoelectric element, which is larger than any other characteristic time of the system. We will employ a connecting function of the form $F(p, V)=k_{c} p$, where $k_{c}$ is the coupling constant of the piezoelectric sample; nonlinear processing of the voltage delivered by the piezoelectric material in order to enhance the electromechanical conversion has been previously considered [24], but is out of the scope of the objectives of the present work. Since our goal is to understand the effect of the nonlinear damping on the performance of the device we have chosen simplest, i.e. linear, expression for the connecting function in the analysis below.

In this work we are considering a Ornstein-Uhlenbeck (OU) random force, with mean $\langle\eta\rangle=0$ and variance $\left\langle\eta(t) \eta\left(t^{\prime}\right)\right\rangle=\sigma \exp \left(-\left|t-t^{\prime}\right| / \tau_{c}\right)$, where $\sigma$ is the amplitude and $\tau_{c}$ is the correlation time. The limit $\tau_{c} \rightarrow 0$ and $\sigma \rightarrow \infty$, with $D=\sigma \tau_{c}$ constant, corresponds to the white noise limit. In order to obtain a closed system of equations, it is a standard procedure to employ the equation $\dot{\eta}=-\eta / \tau_{c}+\bar{\xi}(t) / \tau_{c}$, where $\bar{\xi}(t)$ is a Gaussian white noise with zero mean and correlation $\left\langle\bar{\xi}(t) \bar{\xi}\left(t^{\prime}\right)\right\rangle=2 \sigma \tau_{c} \delta\left(t-t^{\prime}\right)$. Therefore the complete set of equations reads as

$$
\begin{aligned}
\dot{q} & =\frac{p}{m} \\
\dot{p} & =-\omega_{0}^{2} q-\alpha q^{3}-\left(\gamma+\beta q^{2}\right) p-k_{v} V+\eta \\
\dot{V} & =k_{c} p-\frac{1}{\tau_{p}} V \\
\dot{\eta} & =\frac{\eta}{\tau_{c}}+\sqrt{\frac{2 \sigma}{\tau_{c}}} \xi,
\end{aligned}
$$

with a Gaussian white noise correlation of $\left\langle\xi(t) \xi\left(t^{\prime}\right)\right\rangle=$ $\delta\left(t-t^{\prime}\right)$.

The simulations are performed by solving numerically the Langevin equations (2) by using the so-called Heun algorithm; trajectories are computed over an interval of $4 \times 10^{4}$ time units after a transient of $10^{3}$ starting from a set of initial conditions given by $\left\{q(0)=p(0)=0, V(0) \equiv V_{0}=1\right\}$. An ensemble average over $10^{4}$ independent realizations has been performed for each set of parameters.

Being $V^{2}(t) / R_{L}$ the instantaneous power delivered to the load resistance, the measure of performance will be the efficiency (as defined in [18])

$$
\eta_{\mathrm{e}}=\eta_{\mathrm{me}} \eta_{\mathrm{nm}}=\frac{\left\langle V^{2}\right\rangle / R_{L}}{\langle p \eta\rangle / m},
$$

where $\langle\cdots\rangle$ implies both a time-average during the observation interval and an ensemble average over noise realizations, $\eta_{\mathrm{m} e}$ is the transducer's efficiency of converting mechanical 


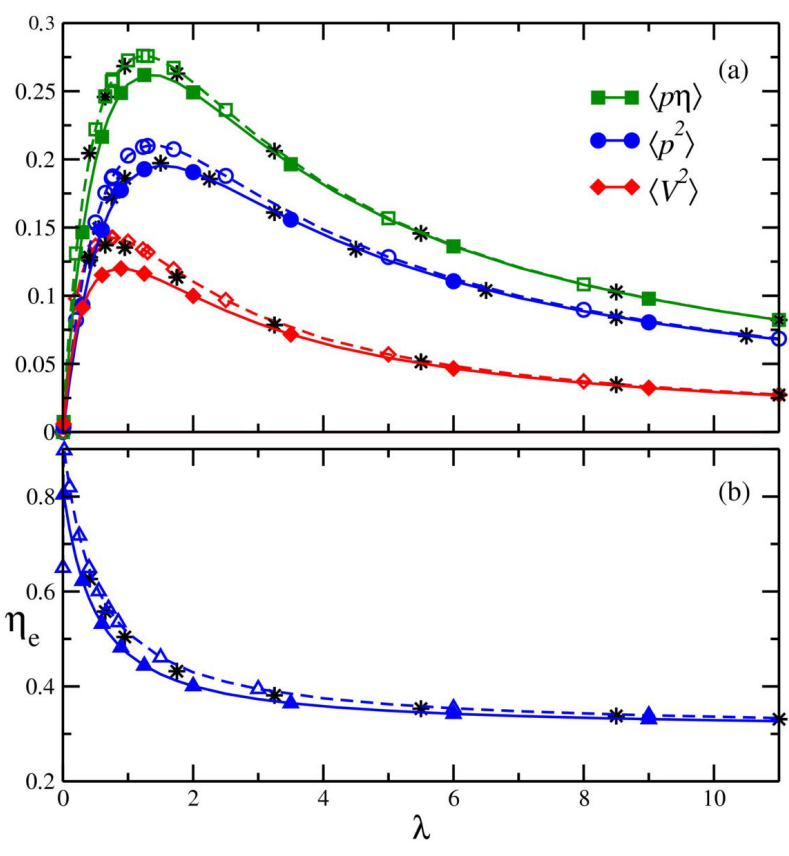

FIgURE 1. (Color online) (a) Comparison of the correlations $\langle p \eta\rangle$ (squares), $\left\langle p^{2}\right\rangle$ (circles), and $\left\langle V^{2}\right\rangle$ (diamonds) computed for the original model (void symbols) and with the nonlinear damping terms (filled symbols). (b) Efficiency $\eta_{\mathrm{e}}$ vs $\lambda$ for the original model (void symbols) and for the one with nonlinear damping terms (filled circles). $\sigma=\gamma=\omega_{0}=m=k_{c}=k_{v}=V_{0}=1 ; \mathcal{C}=\tau_{p}=2$; $\alpha=0, \beta=50$. Asterisks in both graphs correspond to the $\alpha=-10$ value for each curve.

to electrical power, and $\eta_{\mathrm{nm}}$ is the efficiency of power converted from the external noise to the power transferred from the oscillator to the transducer. The explicit expressions are reported in Ref. [18].

\section{Results}

In Fig. 1(a) we present the comparison of the correlations $\langle p \eta\rangle,\left\langle p^{2}\right\rangle$, and $\left\langle V^{2}\right\rangle$ as a function of the inverse of the correlation time $\lambda \equiv \tau_{c}^{-1}$ for the original case reported in [18], i.e. $\alpha=\beta=0$ and for $\alpha=0, \beta=50$; the curves corresponding to $\alpha=\beta=50$ (not shown) are indistinguishable to those reported in the above figure. Just as in the original linear damping case, the electrical power $P$ passes through a maximum, $P^{*}$, as $\lambda$ increases, whereas the efficiency decreases monotonically as $\lambda$ increases. These results are reported with large $\alpha$ and $\beta$ values because only in this range any appreciable difference can be observed in comparison with the linear friction case. Furthermore, these differences are only significant in the region of the maximum of each curve, being negligible as $\lambda$ increases. The net effect is to reduce the height of each curve, thus reducing the maximum power compared to the original instance, and to shift the location of the maximum to a larger $\lambda$ value. The net result, as can be appreciated in Fig. 1(b), is a reduced efficiency for all studied values of the inverse of the correlation time, as could be expected of the dissipative nature of the newly considered terms. We also performed further simulations with the $\alpha=-10$ value and we can readily appreciate in Fig. 1(a) that the correlation $\left\langle p^{2}\right\rangle$ is largely insensitive to the change in sign in the intensity of the Duffing force. However the remaining correlations present a rather amusing behavior: they are almost the same as those corresponding to the linear friction case. This phenomenology results in an efficiency that is also very similar to that of the linear damping case, as can be seen in Fig. 1(b). A possible explanation could be inferred from the fact that the shape of the potential corresponding to the conservative forces of the system with $\alpha=-10$ in the region close to the origin, i.e. for moderate displacements, is very similar to the one corresponding to $\alpha=10$, a value wherewith the efficiency of the system with a nonlinear damping is indistinguishable from the one obtained with a system without it. This result suggest that, if the nonlinear damping becomes relevant in any particular instance, its negative effects in the efficiency of the device could potentially be diminished with the inclusion of a Duffing force of negative amplitude.

Since in both considered instances the maximum efficiency does not occur when the net electrical power is maximal and vice versa, we studied how the maximum net electrical power $P^{*}$, the efficiency at the maximum power $\eta_{\mathrm{e}}^{*}$, and the characteristic frequency of the noise at the maximum power $\lambda^{*}$ depend on the parameters $\tau_{p}, k_{c}, k_{v}$, and $\omega_{0}$ of the energy harvester. Of these parameters the first three are related to the electrical circuit of the transducer and the last one characterizes the mechanical behavior of the mass-spring system. In each case the considered parameter is varied and the rest are taken equal to 1 . Considering the dependence with respect to $\tau_{p}$ presented in Fig. 2(a) $\lambda^{*}$ decreases mono-
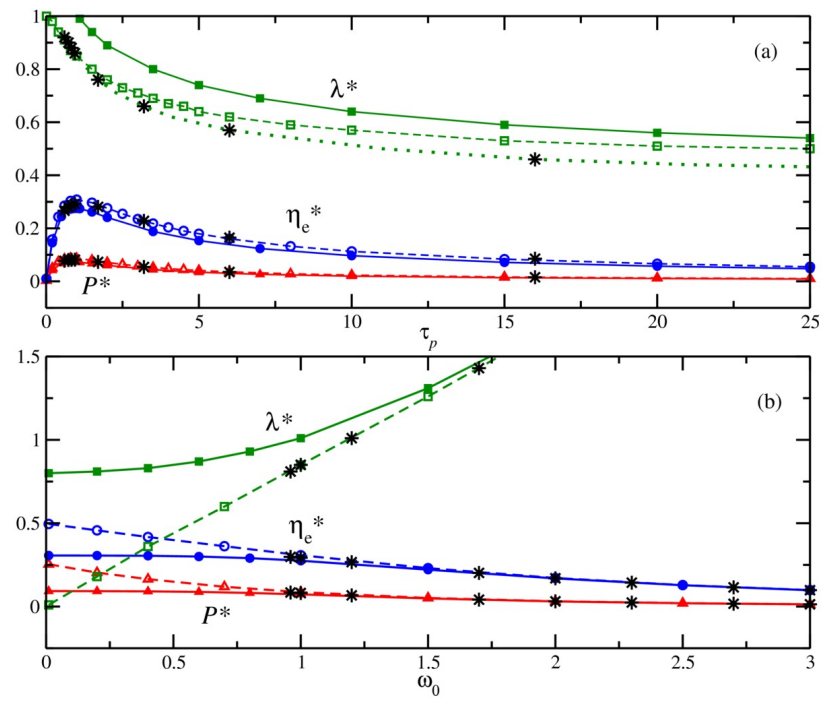

FIGURE 2. (Color online) Maximum net electrical power $P^{*}$, efficiency at the maximum power $\eta_{\mathrm{e}}^{*}$, and characteristic frequency of the noise at the maximum power $\lambda^{*}$ as a function of (a) $\tau_{p}$ and (b) $\omega_{0}$. In each panel we vary the corresponding parameter, set all other ones equal to 1 , and take $\mathcal{C}=1$. Same $\alpha$ and $\beta$ values as in Fig. 1. Asterisks in both graphs correspond to the $\alpha=-10$ value for each curve. 

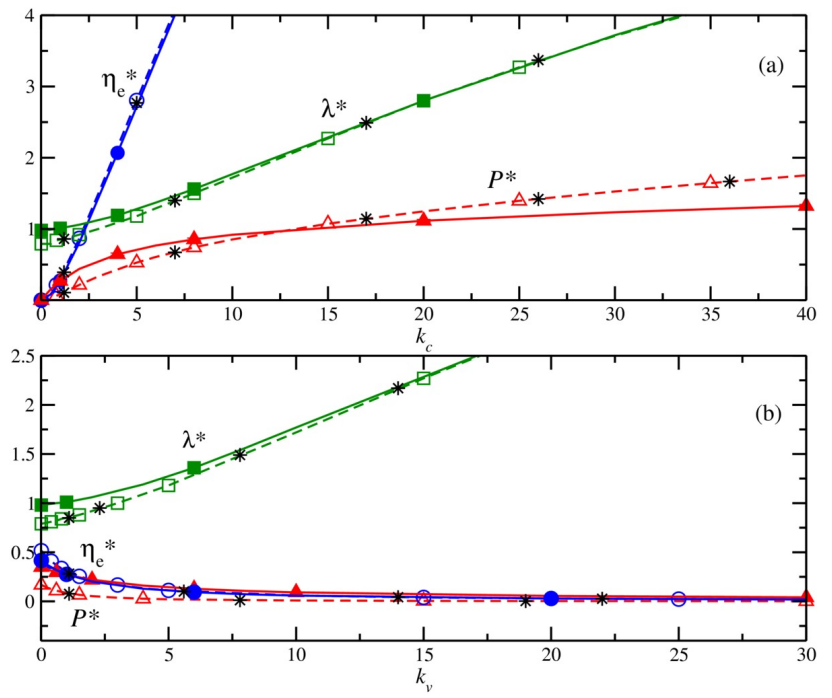

FIgURE 3. (Color online) $P^{*}, \eta_{\mathrm{e}}^{*}$, and $\lambda^{*}$ as a function of (a) $k_{c}$ and (b) $k_{v}$. In each panel we vary the corresponding parameter and set all other ones equal to 1 , with $\mathcal{C}=1$. Again, same $\alpha$ and $\beta$ values as in Fig. 1. Asterisks in both graphs correspond to the $\alpha=-10$ value for each curve.

tonically as $\tau_{p}$ increases in both instances, with a larger decay for the system with nonlinear friction, whereas $P^{*}$ remains almost constant in both instances for the considered variation. In contrast, $\eta_{\mathrm{e}}^{*}$ increases for small $\tau_{p}$ values, reaches a maximum and then decreases monotonically as $\tau_{p}$ further increases; the maximum is smaller for the system with nonlinear damping. Therefore it is convenient to take small $\tau_{p}$ values to optimize the efficiency for both types of damping. With the value $\alpha=-10$ the decay of $\lambda^{*}$ is even faster than that corresponding to the system with linear damping, but otherwise the behavior of the other two variables is very similar to that of the system without nonlinear damping. The behavior of $P^{*}$ and $\eta_{\mathrm{e}}^{*}$ as the frequency $\omega_{0}$ changes is different in both instances, as can be inferred from the results reported in Fig. 2(b). For the linear damping case both variables monotonically decrease as $\omega_{0}$ increases, whereas in the case with nonlinear damping the behavior of both variables is different of the aforementioned one at small frequencies, i.e. both are weakly dependent on the frequency for $\omega_{0}<1$ and then slowly decreases for $\omega_{0}>1$. Thus in this case it is convenient to take small $\omega_{0}$ values to optimize the maximum net electrical power and the efficiency at maximum power. For $\alpha=-10$ we could only compute values in the $\omega_{0}>1$ range, and these are the same as those for the system without the nonlinear damping term. Therefore, in this case taking the lower bound $\omega_{0}=1$ would be the only available choice to optimize both $P^{*}$ and $\eta_{\mathrm{e}}^{*}$.

The variation with respect to $k_{c}$, shown in Fig. 3(a), indicates that $\eta_{\mathrm{e}}^{*}$ significantly increases for low $k_{c}$ in both instances, with minimal differences between them. On the other hand, $P^{*}$ also increases for low $k_{c}$ values in both cases, being greater for the device including the nonlinear friction term if $k_{c}<10$, but rapidly approaches an asymptotic value for $k_{c}>10$, unlike the case with only a linear friction term, which keeps increasing in this value range. Thus, and considering that the physically acceptable regime corresponds to $\eta_{\mathrm{e}}^{*}<1$, the value $k_{c} \sim 2.5$ can be considered as an acceptable compromise to optimize the performance of the device when the nonlinear damping is relevant. In the case in which the variation with respect to $k_{v}$ is considered, depicted in Fig. 3(b), the behavior of $\eta_{\mathrm{e}}^{*}$ is virtually identical in both considered cases. However, the maximum net electrical power $P^{*}$ has a higher value in the low $k_{v}$ range for the nonlinear damping case compared to the one with linear damping. Therefore a value of $k_{v} \sim 1$ is adequate to optimize the values of both the maximum net electrical power and the efficiency at the maximum power when the nonlinear damping is relevant. For the $\alpha=-10$ value the results in both panels (a) and (b) are virtually the same to those for the corresponding cases with linear damping.

Summarizing the previous discussion, the characteristic frequency of the noise at the maximum power $\lambda^{*}$ decreases monotonically as $\tau_{p}$ increases, see Fig. 2(a), whereas it increases monotonically as $k_{v}$ and $k_{c}$ increase, as depicted in Figs. 3(a) and (b). Special mention deserves the dependence of $\lambda^{*}$ with respect to $\omega_{0}$ presented in Fig. 2(b), which is very weak for low $\omega_{0}$ values, precisely in the value range wherein both $P^{*}$ and $\eta_{\mathrm{e}}^{*}$ are almost independent of the natural frequency of the oscillator. In this case our result suggest to take a $\omega_{0}$ value no larger than 0.5 to optimize the performance of the device, except when $\alpha=-10$ is used, in which case $\omega_{0}=1$ has to be taken for the reasons exposed above. In all considered instances the $\lambda^{*}$ values are higher for the case with nonlinear damping compared to the one with linear damping. Therefore, we can conclude that to improve performance it would be desirable to take $\tau_{p}, \omega_{0}<1$ ( $\omega_{0}=1$ with $\alpha=-10$ ) for both cases, with and without nonlinear damping. As for the remaining parameters the best value would be at around $k_{v}, k_{c} \sim 2.5$ since for this value the efficiency is optimized and the maximum net electrical power $P^{*}$ reaches a value that is still significant compared to the one obtained with the tuning of the remaining parameters.

\section{Discussion and conclusions}

Our results on the performance of a nonlinear electromechanical oscillator with a nonlinear coupling with an external finite-bandwidth ambient noise seem to indicate that the performance of the system as an energy harvester is only weakly affected by the presence of the aforementioned nonlinear coupling, since the constants that define the latter have to take exceedingly large values in order to affect in a mensurable way the correlations that characterize the performance of the considered energy harvester. In particular, the correlations wherewith both the maximum power and efficiency are defined present a very weak dependence on the nonlinear parameters of the interaction with the source of the ambient noise. 
A constant among the proposed energy-harvesting devices is that the combined goals of both maximum power and efficiency cannot be attained at the same time, being the present one no exception. A compromise in the value of all parameters has to be made, and thus we determined that the best performance of the energy harvester is obtained by taking low values for the characteristic charging time of the capacitor $\tau_{c}$ and the piezoelectric parameter $k_{v}$ to obtain the maximum values of both $P^{*}$ and $\eta_{\mathrm{e}}^{*}$ in each instance. For $k_{c}$ an intermediate value allows to obtain a significant value for the maximum net electrical power together with a high efficiency at maximum power. The characteristic frequency of the mechanical oscillator has to take values of $\omega_{0} \sim 0.5$ (and $\omega_{0}=1$ for the case $\alpha=-10$ ) to take advantage of the fact that in this value range the maximum power, the maximum efficiency at maximum power as well as the characteristic fre- quency at maximum power have an approximately constant value. A positive amplitude of the Duffing force has no effect on the performance of the device and, with a negative amplitude, it was possible to minimize the adverse effects of the nonlinear damping in the efficiency of the device. In view of this finding it would be interesting to study the performance of an energy harvester affected by the herein studied nonlinear coupling with the external noise source but employing a bistable symmetric potential, which has gained attention recently, as already mentioned [9-12]. We intend to investigate this particular possibility in a future work.

\section{Acknowledgments}

M. R. B. gratefully acknowledges CONACyT, México for financial support.
1. S. P. Beeby, M. J. Tudor, and N. M. White, Measurement Science and Technology 17 (2006) R175.

2. S. R. Anton and H. A. Sodano, Smart Materials and Structures 16 (2007) R1.

3. J. M. Renno, M. F. Daqaq, and D. J. Inman, Journal of Sound and Vibration 320 (2009) 386.

4. A. Cammarano et al., Smart Materials and Structures 19 (2010) 055003 .

5. H. S. Kim, J.-H. Kim, and J. Kim, International Journal of Precision Engineering and Manufacturing 12 (2011) 1129.

6. P. D. Mitcheson et al., Proceedings of the IEEE 96 (2008) 1457.

7. D. Spreemann and Y. Manoli, Electromagnetic Vibration Energy Harvesting Devices: Architectures, Design, Modeling and Optimization (Springer, Dordrecht, 2012).

8. S. G. Burrow, L. R. Clare, A. Carrella, and D. Barton, in Proceedings of the SPIE smart structures/NDE conference, edited by M. Ahmadian (SPIE, San Diego, California, USA, 2008).

9. F. Cottone, H. Vocca, and L. Gammaitoni, Phys. Rev. Lett. 102 (2009) 080601.

10. L. Gammaitoni, I. Neri, and H. Vocca, Appl. Phys. Lett. 94 (2009) 164102.

11. A. Erturk, J. Hoffmann, and D. J. Inman, Appl. Phys. Lett. 94 (2009) 254102.
12. H. Vocca, I. Neri, F. Travasso, and L. Gammaitoni, Applied Energy 97 (2012) 771.

13. M. F. Daqaq, Journal of Sound and Vibration 329 (2010) 3621.

14. P. L. Green, K. Worden, K. Atallah, and N. D. Sims, Journal of Sound and Vibration 331 (2012) 4504.

15. E. Halvorsen, Phys. Rev. E 87 (2013) 042129.

16. S. D. Nguyen and E. Halvorsen, Journal of Microelectromechanical Systems 20 (2011) 1225.

17. M. F. Daqaq, Journal of Sound and Vibration 330 (2011) 2554.

18. V. Méndez, D. Campos, and W. Horsthemke, Phys. Rev. E 88 (2013) 022124.

19. A. Eichler et al., Nat. Nanotech. (2011) 339.

20. D. Segal and A. Nitzan, Phys. Rev. Lett. 94 (2005) 034301.

21. D. Segal and A. Nitzan, J. Chem. Phys. 122 (2005) 194704.

22. D. Segal, Phys. Rev. Lett. 100 (2008) 105901.

23. Y. Ming, H.-M. Li, and Z.-J. Ding, Phys. Rev. E 93 (2016) 032127.

24. E. Lefeuvre et al., Sensors and Actuators A: Physical 126 (2006) 405. 